\title{
Quantitative Methods for Prediction of the Effect of Cytochrome P450 Gene Polymorphisms on Substrate Drug Exposure: Authors' Reply
}

\author{
Jonatan D. Lindh · Ming Chang • Gunnel Tybring • \\ Marja-Liisa Dahl
}

Published online: 9 December 2014

(c) Springer International Publishing Switzerland 2014

We would like to thank Goutelle and Tod for their interest in our study and for bringing their previous publication on cytochrome P450 2C19 (CYP2C19) polymorphisms to our attention [1]. First, we would like to clarify that we have not claimed our study to be the first to provide precise estimates of the influence of CYP2C19 genotypes on (es)citalopram exposure, but the first to quantify this influence by means of a systematic review and meta-analysis [2]. We still believe this holds true and would like to point out a few pivotal differences between our metaanalysis and the study by Goutelle et al. Most importantly, our analysis was based on a comprehensive literature review to assure that the estimates took into account all available pharmacokinetic data, while their analysis was based on a limited number of studies assembled in a fashion that was not described in detail. In addition, we restricted our scope to studies on (es)citalopram, accounting for the possibility that the impact of CYP2C19 polymorphisms may differ between different CYP2C19 substrates. In contrast, the analysis by Goutelle and coworkers was based on studies of a large number of CYP2C19 substrates, implicitly assuming that the clearance attributable to CYP2C19 would be affected equally

J. D. Lindh · M. Chang $(\bowtie) \cdot$ M.-L. Dahl

Division of Clinical Pharmacology, Department of Laboratory

Medicine, Karolinska Institutet, Karolinska University Hospital,

Stockholm, Sweden

e-mail: ming.chang@ki.se

\section{Chang}

Exploratory Clinical and Translational Research, Bristol-Myers

Squibb Company, Pennington, NJ 08534, USA

\section{G. Tybring}

Department of Medical Epidemiology and Biostatistics,

Karolinska Institutet, Stockholm, Sweden for all substrates. Only two of these studies provided data specifically concerning citalopram, as compared with the 14 publications included in our meta-analysis. The fact that both methods produced similar results is reassuring, because it confirms both the robustness of the pharmacogenetic effect estimates and the usefulness of the unified modeling approach proposed by Ohno et al. [3] and Tod et al. [4].

\section{References}

1. Goutelle S, Bourguignon L, Bleyzac N, Berry J, Clavel-Grabit F, et al. In vivo quantitative prediction of the effect of gene polymorphisms and drug interactions on drug exposure for CYP2C19 substrates. Aaps J. 2013;15:415-26.

2. Chang M, Tybring G, Dahl ML, Lindh JD. Impact of cytochrome P450 2C19 polymorphisms on citalopram/escitalopram exposure: a systematic review and meta-analysis. Clin Pharmacokinet. 2014;53:801-11.

3. Ohno Y, Hisaka A, Suzuki H. General framework for the quantitative prediction of CYP3A4-mediated oral drug interactions based on the AUC increase by coadministration of standard drugs. Clin Pharmacokinet. 2007;46:681-96.

4. Tod M, Goutelle S, Gagnieu MC. Genotype-based quantitative prediction of drug exposure for drugs metabolized by CYP2D6. Clin Pharmacol Ther. 2011;90:582-7. 